\title{
Évaluation dosimétrique et biogénotoxicologique de l'exposition aux rayonnements ionisants
}

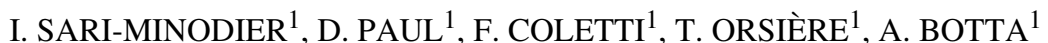

(Manuscrit reçu le 6 novembre 2006, accepté le 28 février 2007)

RÉSUMÉ Le Laboratoire de Biogénotoxicologie et Mutagenèse Environnementale a pour objectifs la détection précoce des effets génotoxiques des nuisances environnementales, la validation de biomarqueurs et la biosurveillance de salariés exposés à un environnement professionnel potentiellement cancérogène. Les travaux menés par le laboratoire dans le domaine des rayonnements ionisants, associant mesures environnementales et évaluation de la génotoxicité dans un objectif de prévention, concernent les travailleurs exposés en radiographie industrielle et en milieu hospitalier. La caractérisation des expositions repose ainsi sur l'analyse des activités avec dosimétrie physique, le recueil des enregistrements dosimétriques et la mise en œuvre de biomarqueurs d'effets génotoxiques tels que le test des micronoyaux sur lymphocytes, permettant de détecter des remaniements chromosomiques de structure ou de nombre. Les données d'analyse de poste associées à des modélisations ont également permis d'établir un tableau de doses de référence, pour chaque étape constitutive d'un tir en gammagraphie industrielle, ce qui constitue un outil intéressant pour la dosimétrie prévisionnelle.

ABSTRACT Dosimetric and genotoxic assessment of occupational exposure to ionizing radiations. The aim of the "Biogénotoxicologie et Mutagenèse Environnementale" Laboratory is the early detection of environment genotoxic effects, the validation of biomarkers and the biomonitoring of workers occupationally exposed to potentially carcinogenic environment. Several studies have been conducted in the field of ionizing radiation, combining environmental exposure and genetic effect measurements in a preventive purpose. They concern industrial radiographers and hospital workers. Exposure is characterized by (i) the analysis of working conditions using physical dosimetry, (ii) the collection of individual dosimetry records and (iii) the application of genotoxicity biomarkers as the micronucleus assay in lymphocytes to assess chromosome breakage or loss. A reference doses table has been established, for different stages of a shooting in industrial gammagraphy, by combining dose measurements and calculations; this constitutes a valuable tool for provisional dosimetry.

Keywords : industrial radiography / medical radiation worker / dosimetry / genotoxicity / biomarker

${ }^{1}$ Université de la Méditerranée, Faculté de Médecine de Marseille, Laboratoire de Biogénotoxicologie et Mutagenèse Environnementale (EA 1784 - IFR PMSE 112), 27 boulevard Jean Moulin, 13385 Marseille Cedex 5, France. 


\section{Introduction}

Le Laboratoire de Biogénotoxicologie et Mutagenèse Environnementale (BME), équipe d'accueil EA 1784, est associé au Service Hospitalo-Universitaire de Médecine et Santé au Travail du CHU de Marseille et intégré dans l'IFR Pôle Méditerranéen des Sciences de l'Environnement. Il a pour objectifs la détection précoce des effets génotoxiques des nuisances environnementales, la validation de biomarqueurs d'exposition, d'effets et de susceptibilité et la biosurveillance de salariés exposés à un environnement professionnel potentiellement cancérogène. Parmi les nuisances environnementales les plus intéressantes au plan scientifique et les plus porteuses d'interrogations au niveau du grand public, les rayonnements ionisants (RI) ont toujours occupé une place privilégiée. En effet, si leur action génotoxique et mutagène est bien documentée dans les cas de fortes expositions, de nombreuses questions d'ordre mécanistique demeurent dans les cas des faibles et très faibles expositions pour lesquelles, en outre, les approches en terme de risque pour la santé sont malaisées. Sans aller jusqu'à évoquer des effets protecteurs par mise en état de « vigilance » des systèmes cellulaires de réparation des lésions de l'ADN (théorie de l'hormesis), on ne peut rien affirmer aujourd'hui quant à la nature exacte, aux mécanismes et aux conséquences des effets génotoxiques des faibles et très faibles doses de RI. C'est pourquoi une des approches nécessaires à développer est la caractérisation des expositions humaines à de telles doses, c'est-à-dire l'expologie. Celle-ci fait appel certes aux biomarqueurs d'exposition et d'effets mais doit intégrer également la dosimétrie physique dans le but de quantifier les niveaux d'exposition externe des individus. L'association dosimétrie-biosurveillance-enquête de terrain pour comprendre les circonstances d'exposition est incontournable pour répondre à la définition la plus pertinente de l'expologie. La collaboration étroite entre préventeurs, analystes et médecins du travail doit donc être effective avant d'envisager toute démarche d'expologie. La présence de spécialistes de la dosimétrie physique au sein du laboratoire BME a ainsi amené un élément complémentaire indispensable à la réalisation des études de caractérisation des expositions professionnelles aux faibles doses de RI.

\section{Méthodes d'évaluation de la génotoxicité des rayonnements ionisants}

Sur le plan fondamental, on sait que les RI sont susceptibles de provoquer de multiples lésions de la molécule d'ADN, dont les plus caractéristiques sont les cassures double brin. Ces dernières, de par leur faible niveau de réparabilité, sont considérées comme les lésions les plus efficaces quant à la production d'effets biologiques. Des lésions de l'ADN non ou mal réparées peuvent entraîner la mort 
de la cellule (effets déterministes) ou être à l'origine de mutations, qui selon leur type, leur nombre, leur situation au niveau du génome, sont susceptibles d'initier le processus long et complexe de cancérogenèse (effets stochastiques). La mise en évidence précoce d'une génotoxicité chez les personnes exposées à des cancérogènes/mutagènes s'inscrit donc bien dans une démarche de prévention des conséquences à long terme sur la santé.

Divers biomarqueurs sont disponibles pour mettre en évidence les effets génotoxiques des RI ainsi que des agents chimiques, qu'il s'agisse de lésions primaires de l'ADN, susceptibles d'être réversibles sous l'effet des systèmes de réparation, ou de mutations, c'est-à-dire des lésions stables et héritables du patrimoine génétique (Orsière et De Méo, 2005 ; Orsière et al., 2000). On distingue trois types de mutations, les mutations géniques (intéressant quelques paires de bases), chromosomiques (altérations structurales des chromosomes) et génomiques (anomalies du nombre des chromosomes).

\subsection{Détection des lésions primaires de l'ADN}

Il existe différents tests permettant de détecter des lésions de l'ADN, qu'elles soient réparables fidèlement ou de façon fautive par les systèmes enzymatiques de réparation. Il s'agit principalement du test des comètes, du test de synthèse non programmée de l'ADN, du test des échanges de chromatides sœurs et de la détection des dommages oxydatifs de l'ADN par différentes techniques. Pour la mise en évidence de ces lésions primaires de l'ADN, le laboratoire BME utilise principalement le test des comètes et le dosage de la 8-hydroxydeoxyguanosine (8-OHdG).

\subsubsection{Test des comètes}

Le test des comètes, technique microélectrophorétique rapide et sensible, permet de visualiser les lésions primaires de l'ADN, à savoir les cassures simple et double brin, les sites labiles alcalins, les pontages et les sites incomplets de réparation. Après traitement et lyse des cellules, une courte électrophorèse suivie d'une coloration fait apparaître l'ADN d'une cellule intacte sous la forme d'une sphère alors que l'ADN d'une cellule lésée a un aspect « en comètes ». Ce test est de plus en plus utilisé dans des applications in vitro, notamment pour étudier des cinétiques de réparation (Decome et al., 2005), ou après exposition in vivo (Botta et al., 2006 ; Iarmarcovai et al., 2005). Les applications en biosurveillance nécessitent des précautions particulières du fait de la très grande sensibilité de ce biomarqueur au stress oxydatif notamment. Son intérêt est également de pouvoir évaluer les capacités de réparation de l'ADN après irradiation in vitro de lymphocytes de sujets exposés professionnellement ou non aux RI. 


\subsubsection{Dosage de la 8-hydroxydeoxyguanosine}

La 8-OHdG est le produit d'oxydation de l'ADN le plus abondant et a un potentiel mutagène relativement important. Cet adduit à la guanine fait partie des dommages de bases susceptibles d'être provoqués par le stress oxydatif induit par les RI par l'intermédiaire de la radiolyse de l'eau. Il est possible de doser la 8-OHdG, éliminée dans les urines après réparation de l'ADN, par des techniques de chromatographie liquide haute performance ou, plus récemment, par des techniques ELISA.

\subsection{Détection des mutations}

Les principaux tests de mutations géniques appliqués aux RI sont ceux détectant des mutations sur le locus hypoxanthine-guanine phosphoribosyl transférase (hprt) ou sur le locus glycophorine A. Quant à la détection des mutations chromosomiques, elle est effectuée par le test des aberrations chromosomiques et le test des micronoyaux (MN), ce dernier permettant également de mettre en évidence les mutations génomiques.

\subsubsection{Test des aberrations chromosomiques}

Les cassures double brin de la molécule d'ADN non ou mal réparées se traduisent par des anomalies structurales des chromosomes visibles en métaphase. On distingue les aberrations dites instables (dicentriques, anneaux, fragments acentriques) et celles dites stables (inversions, translocations) qui se transmettent au cours des divisions cellulaires successives.

Le test des aberrations chromosomiques repose sur l'obtention de préparations chromosomiques métaphasiques après blocage de la mitose in vitro. Les chromosomes sont alors observés au microscope et une analyse visuelle qualitative et quantitative est effectuée. Les méthodes cytogénétiques conventionnelles permettent d'enregistrer les délétions importantes ainsi que les réarrangements complexes, tandis que la mise en évidence des translocations nécessite des techniques plus complexes telles que l'hybridation in situ fluorescente (FISH) réalisant une véritable peinture chromosomique.

La numération des aberrations chromosomiques est une des méthodes les plus anciennes et les plus utilisées dans le domaine des RI. Elle constitue la méthode de référence pour la dosimétrie biologique mise en œuvre après un accident d'irradiation, cette dernière reposant classiquement sur le dénombrement des chromosomes en anneaux et des chromosomes dicentriques dans les lymphocytes périphériques. De nombreuses études ont été conduites avec ce test auprès de 
populations professionnellement exposées aux RI, notamment dans le secteur médical et l'industrie nucléaire.

\subsubsection{Test des micronoyaux}

Le test des $\mathrm{MN}$ a été initialement proposé comme une alternative simple à la numération des aberrations chromosomiques et applicable à la biosurveillance de populations importantes. Les MN sont des entités nucléaires surnuméraires et de petite taille qui contiennent des fragments chromosomiques acentriques, consécutifs à des cassures double brin de l'ADN, et/ou des chromosomes entiers non intégrés dans les noyaux fils au cours de la mitose du fait d'altérations de l'appareil mitotique. La numération des MN représente ainsi un moyen fiable d'évaluer simultanément les mutations chromosomiques et génomiques, consécutives respectivement à des évènements clastogènes ou aneugènes. On appelle évènements clastogènes ceux qui procèdent par des cassures chromosomiques (cassures double brin de la molécule d'ADN) et évènements aneugènes ceux qui procèdent par la perte de chromosomes entiers du fait de l'altération de l'appareil mitotique (liée principalement à des interactions avec les protéines).

Le test des MN, réalisé le plus souvent sur des lymphocytes périphériques dans le cadre des études de biosurveillance des populations exposées, recourt à une mise en culture des cellules avec blocage de la division cytoplasmique, d'où l'obtention de lymphocytes binucléés permettant d'identifier sans ambiguïté les cellules porteuses d'altérations de type cassure double brin ou perte de chromosomes entiers. En effet, ces anomalies ne se traduisent par la formation d'un $\mathrm{MN}$ qu' au cours d'une mitose complète. L'observation au microscope permet de dénombrer la présence de $\mathrm{MN}$ au sein des lymphocytes binucléés. Les résultats sont exprimés en taux de lymphocytes binucléés micronucléés (LBMN), c'est-à-dire contenant un ou plusieurs MN, pour 1000 lymphocytes binucléés. Le couplage de ce test à l'hybridation in situ fluorescente de sondes pancentromériques permet de distinguer les MN contenant un ou plusieurs fragments chromosomiques acentriques ( $\mathrm{MN}$ centromère-négatifs sans spot de fluorescence) de ceux contenant un ou plusieurs chromosomes entiers ( $\mathrm{MN}$ centromère-positifs avec un ou plusieurs spots de fluorescence).

Le test des MN fait l'objet depuis quelques années d'une large étude internationale, intitulée The Human MicroNucleus (HUMN) Project. Il a été validé quant à sa capacité à détecter les effets de faibles doses de RI tant in vitro qu'in vivo. Il est employé dans de nombreuses études de biosurveillance, notamment auprès de populations exposées aux RI au niveau environnemental ou professionnel (Sari-Minodier et al., 2007, 2004, 2002, 2000 ; Sari-Minodier, 2002). 


\section{3. Évaluation et prévention du risque radiologique professionnel dans les opérations de radiographie industrielle}

\subsection{Introduction}

La radiographie industrielle est l'un des principaux outils pour le contrôle non destructif (CND), notamment pour le contrôle des soudures, pour le repérage des défauts des matériaux et pour évaluer la corrosion. Les radiologues industriels comptent parmi les travailleurs les plus exposés aux RI. Leur dosimétrie individuelle élevée est due à des conditions de travail difficiles. En 1996, une charte de bonnes pratiques a été rédigée par les opérateurs et les administrations publiques pour la région Provence-Alpes-Côte d'Azur (PACA).

Il apparaît actuellement un regain d'intérêt pour la radioprotection des radiologues industriels au regard de la nouvelle réglementation basée sur la perception et la gestion du risque d'exposition. Le principal objectif de l'étude en cours (2004-2006), coordonnée par le laboratoire BME, est la caractérisation approfondie de l'exposition professionnelle et l'application du principe d'optimisation avec une charte de bonnes pratiques mise à jour. En 2003, les chiffres fournis par l'Institut de radioprotection et de sûreté nucléaire (IRSN) révèlent que sept salariés travaillant dans l'industrie ont reçu entre 20 et $50 \mathrm{mSv}$ et trois ont dépassé la limite autorisée de $50 \mathrm{mSv}$ à cette époque.

L'exposition a été caractérisée suivant deux approches complémentaires : l'analyse des conditions de travail à partir de la dosimétrie physique et la surveillance dosimétrique et biogénotoxicologique. Un tableau de doses de référence a été élaboré pour chaque étape constitutive d'un tir durant des opérations de routine. Ce tableau est issu de données expérimentales (dosimétrie opérationnelle) et de calculs (modélisation). Il servira de repère pour aider les radiologues industriels à faire une dosimétrie prévisionnelle comme l'exige la réglementation selon le principe ALARA. L'analyse des postes de travail et des enquêtes auprès des parties prenantes ont également permis de fournir des données complémentaires pour évaluer l'ensemble des procédures sur les plans administratif, technique et pratique.

La nouvelle charte prend en compte le retour d'expérience de la précédente version, la nouvelle réglementation et les évolutions techniques. Plus conviviale, elle revêt également un caractère plus opérationnel suivant un processus d'amélioration continue de la qualité. 


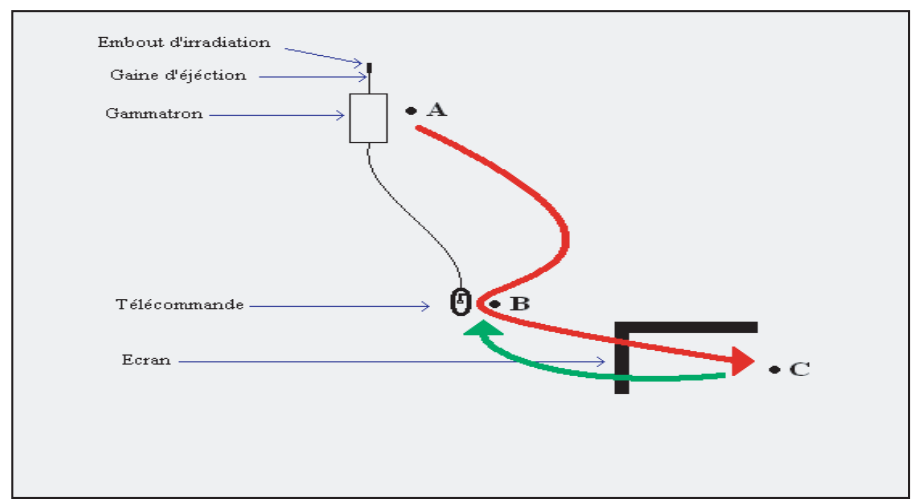

Figure 1 - Parcours effectué par les opérateurs.

Course done by the operators.

\subsection{Matériels et méthodes}

\subsubsection{Analyse des conditions de travail à partir de la dosimétrie physique}

L'étude porte exclusivement sur la radiographie gamma réalisée dans la majorité des cas avec des sources d'iridium-192. L'analyse des conditions de travail du radiologue a consisté dans un premier temps à évaluer la préparation du matériel dans l'entreprise de radiographie puis à prendre en compte le transport du gammagraphe, considéré comme une matière dangereuse, jusqu'au lieu de tirs. Arrive ensuite la séance de tirs proprement dite avec plusieurs éjections de la source par nuit de travail et finalement le retour à l'entreprise pour la lecture et l'interprétation des films.

Les conditions de travail varient considérablement d'un site à l'autre en fonction du type d'application comme les inspections de pipelines, la détection de fuites et la mesure de corrosion dans les centrales nucléaires ou sites pétrochimiques, le travail en atelier ou encore dans des blockhaus.

Le matériel utilisé par notre laboratoire pour les mesures est celui de l'instrumentation de radioprotection classique; il comporte deux appareils de mesure d'ambiance basés sur une technologie Geiger Müller et un dosimètre individuel électronique pour la surveillance individuelle. Le protocole de mesure a été mis au point au laboratoire (Paul et al., 2006 ; Azzopardi et al., 2005 ; Azzopardi, 2005) avec différents points de mesure de l'équivalent de dose ou du débit d'équivalent de dose ambiant. Ces points sont répartis sur le trajet du radiologue comme le montre la figure 1. Cette figure reproduit le déroulement d'un tir, de l'éjection à la rentrée de la source (points $\mathrm{A}, \mathrm{B}$ et $\mathrm{C}$ ). 
L'étape du point $\mathrm{A}$ au point $\mathrm{C}$ représente l'équivalent de dose cumulé lors des opérations suivantes: ouverture de l'obturateur, déplacement jusqu'à la télécommande (trajet $\mathrm{AB}$ ), éjection de la source, repli de l'opérateur (trajet $\mathrm{BC}$ ). L'étape du point $C$ au point $B$ représente l'équivalent de dose reçu lors du déplacement jusqu'à la télécommande et la rentrée de la source avec fermeture automatique de l'obturateur.

Des calculs complémentaires ont été faits par le laboratoire (Coletti et al. 2006 ; Pizzorno et al. 2006 ; Klemenic, 2005) avec le code Mercurad, version 6.3 (licence CEA et COGEMA), Canberra Eurisys S.A., pour simuler ses équivalents de dose dans un cas simple, selon une activité donnée. Le but de ces calculs était de faire des extrapolations pour remonter à la dosimétrie globale pour une séance de tirs et de fournir aux radiologues des éléments de dosimétrie prévisionnelle avec les paramètres d'exposition les plus importants.

\subsubsection{Surveillance dosimétrique et biogénotoxicologique}

La dosimétrie individuelle est réglementée par le code du travail (décret 2003-296 du 31 mars 2003). Ce décret et l'arrêté du 30 décembre 2004 prévoient les caractéristiques requises pour les dosimètres, les modalités de la dosimétrie passive et de la dosimétrie opérationnelle, les limites de dose annuelles, l'enregistrement des doses pour chaque travailleur et le suivi par l'IRSN.

Un radiologue industriel est toujours en catégorie A et porte un film dosimétrique étalonné en équivalent de dose individuel $(\mathrm{Hp}(10))$. Il doit également détenir un dosimètre électronique (dosimètre opérationnel) pour la mesure en temps réel de l'équivalent de dose. Ces dosimètres offrent la possibilité de programmer des contraintes de dose et de débits de dose avec des seuils et une alarme sonore.

Tous les médecins du travail de la région PACA impliqués dans la surveillance de radiologues industriels ont été sollicités pour participer à l'étude menée par le laboratoire BME. L'objectif est d'évaluer chez ces travailleurs la génotoxicité de l'exposition professionnelle aux RI, cette dernière étant documentée par le recueil des enregistrements dosimétriques individuels sur les dix dernières années.

La génotoxicité est évaluée par deux tests complémentaires, le dosage urinaire de la 8-OHdG (avant et après exposition) d'une part, comme marqueur du stress oxydatif, et le test des MN sur lymphocytes d'autre part, comme marqueur d'altérations chromosomiques. Ce dernier test, effectué à partir d'une prise de sang, est couplé à la technique FISH avec sondes pancentromériques afin de distinguer les MN consécutifs respectivement à la perte de fragments chromosomiques acentriques ou de chromosomes entiers. Enfin, des biomarqueurs de susceptibilité 
individuelle, à savoir l'étude du polymorphisme de certains gènes impliqués dans la réponse au stress oxydatif ou dans la réparation des lésions de l'ADN, sont également inclus dans le protocole de biosurveillance, afin d'apporter un éclairage nouveau sur l'interprétation des biomarqueurs d'effets mis en œuvre.

L'ensemble des résultats biologiques est interprété comparativement à ceux obtenus chez des sujets témoins, c'est-à-dire non exposés professionnellement aux RI ou à d'autres nuisances génotoxiques, et appariés à la population de radiologues sur les critères de sexe, âge et tabagisme. L'interprétation tient également compte des données d'exposition basées sur les dosimétries (pour les radiologues) et sur un questionnaire médico-professionnel (pour tous les sujets) destiné également à documenter d'éventuels facteurs de confusion.

\subsubsection{Révision de la charte de bonnes pratiques}

Ces travaux ont permis d'élaborer un projet de nouvelle charte de bonnes pratiques pour la radiographie industrielle (la précédente version date de 1996) afin d'évaluer et prévenir le risque radiologique professionnel dans les opérations de radiographie industrielle. Les donneurs d'ordre et les entreprises de sous-traitance et de radiographie industrielle ont été parties prenantes dans ces travaux.

\subsection{Résultats et discussion}

\subsubsection{Analyse des conditions de travail à partir de la dosimétrie physique}

Un des points importants dans la préparation des tirs est l'évaluation prévisionnelle des doses intégrées par les opérateurs permettant de les optimiser. Dans ce but, un tableau a été élaboré, donnant le calcul des doses aux différentes étapes d'un tir (pour une source d'activité donnée) : transport en voiture du gammagraphe pour se rendre sur le lieu de tir $(1,5 \mu \mathrm{Sv} / \mathrm{h})$, transport manuel et mise en place du gammagraphe $(9,6 \mu \mathrm{Sv} / \mathrm{h}$ au corps entier, $270 \mu \mathrm{Sv} / \mathrm{h}$ aux extrémités) et du collimateur ( $2 \mu \mathrm{Sv} / \mathrm{h}$ au corps entier, $10 \mu \mathrm{Sv} / \mathrm{h}$ aux extrémités), éjection puis retrait de la source radioactive au travers de la gaine d'éjection par action de la manivelle ( $2 \mu \mathrm{Sv} / \mathrm{tir}$ en l'absence d'écran de protection), position de repli durant le temps de tir $(5 \mu \mathrm{Sv} / \mathrm{h})$.

Compte tenu de la très grande diversité des situations de tir et de façon à maximaliser tout en restant réaliste la dose intégrée par les opérateurs, chacune de ces étapes a été modélisée avec le code de calcul «MERCURAD» (version 3) pour une situation où le tir serait fait sans aucun obstacle ou protection. Les mesures dosimétriques faites ont montré que les valeurs obtenues par la modélisation étaient en bon accord (20\%). 
Un logiciel permet ensuite, compte tenu des temps nécessaires pour effectuer chaque étape et du nombre de tirs, d'évaluer les doses intégrées par les opérateurs. Par extrapolation, il est possible d'estimer une dose efficace annuelle. Celle-ci peut dépasser $20 \mathrm{mSv}$ dans les cas les plus défavorables.

\subsubsection{Surveillance dosimétrique et biogénotoxicologique}

À ce jour, les résultats de la biosurveillance génotoxique ne sont pas disponibles, dans la mesure où l'inclusion des sujets n'est pas encore terminée. L'analyse des dosimétries des premiers radiologues industriels inclus dans l'étude ne met pas en évidence de dépassements de la nouvelle limite réglementaire de $20 \mathrm{mSv}$ sur 12 mois (décret 2003-296 du 31 mars 2003), alors que plusieurs enregistrements supérieurs à cette dose étaient notés jusqu'en 2003.

Dans une étude précédemment menée dans le laboratoire BME auprès de 29 radiologues industriels, la dose cumulée moyenne sur la période de 5 ans [19952000] précédant le prélèvement sanguin était de $67 \mathrm{mSv} \pm 50$; sur cette période, 19 sujets $(66 \%)$ présentaient au moins une dose annuelle supérieure $20 \mathrm{mSv}$ et la limite réglementaire de l'époque ( $50 \mathrm{mSv} / \mathrm{an})$ avait été dépassée deux fois. Le test des MN révélait un taux de LBMN significativement supérieur $(10,7 \%$ \pm 5,2 versus $6,6 \% \circ \pm 3,1 ; \mathrm{p}=0,009)$ chez les radiologues comparativement aux témoins $(\mathrm{n}=24)$. L'analyse en FISH a permis de rapporter cette différence à une augmentation de la proportion des MN contenant des fragments chromosomiques ( $74 \%$ chez les radiologues versus $29 \%$ chez les témoins ; $p<0,001$ ), ce qui est en accord avec l'effet clastogène prédominant des RI (Sari-Minodier et al., 2004, 2002 ; Sari-Minodier 2002).

\subsubsection{Révision de la charte}

Cette étude a permis de prendre conscience que les conditions de travail des radiologues industriels doivent être améliorées en respectant les principes de la radioprotection. Les opérations réalisées par un radiologue devront donc être évaluées conformément à la charte de bonnes pratiques signée par les donneurs d'ordre.

Cette charte vise la réduction des expositions par la prise en compte des grands principes de la radioprotection (justification, optimisation et limitation) ainsi que l'amélioration des conditions de travail et l'aide au respect de la réglementation. Elle présente un caractère opérationnel par la prise en compte de la chronologie des différentes étapes du déroulement d'un chantier, en fonction aussi de la nature de ce dernier (site industriel ou atelier). Après un rappel des grands principes de la radioprotection, du rôle et des responsabilités des divers acteurs, la formation $\mathrm{du}$ 
personnel et le suivi médical et dosimétrique des salariés sont abordés. Enfin, le déroulement du chantier est exposé (pré-requis nécessaires à la préparation des travaux, coordination de la prévention, organisation des travaux, transport des sources, déroulement du chantier et retour d'expérience). Des annexes donnent divers détails.

L'Autorité de sûreté nucléaire a défini la protection en radiographie industrielle comme une priorité. Il est envisagé de «nationaliser » cette démarche par l'intermédiaire de la COFREND (Confédération française pour les essais non destructifs) mandatée par la DGSNR avec l'aide de la SFRP (Société française de radioprotection). Ces deux organismes ont pour objectif la rédaction d'un guide national de bonnes pratiques prenant en compte les résultats obtenus dans la région PACA.

\section{4. Évaluation dosimétrique et biogénotoxicologique de l'exposition du personnel hospitalier}

\subsection{Introduction}

Durant les années 2000-2002, dans le contexte d'abaissement annoncé des valeurs limites réglementaires et de mise en place de la dosimétrie opérationnelle, nous avons conduit des études auprès du personnel soignant de l'Assistance Publique des Hôpitaux de Marseille (AP-HM) exposé aux RI avec les objectifs suivants :

- caractériser l'exposition aux RI de différentes catégories de personnel, au sein de divers services hospitaliers, par des études de poste associées à une dosimétrie physique ;

- fournir ainsi des données objectives pouvant servir de base au classement du personnel en catégorie A ou B ;

- analyser les dosimétries passives enregistrées sur les dix dernières années ;

- associer à la caractérisation des expositions une approche biologique par la mise en œuvre du test des MN sur lymphocytes périphériques.

\subsection{Matériels et méthodes}

\subsection{1. Étude des postes de travail avec dosimétrie physique}

Ces études de poste ont été menées en collaboration avec la Cellule de Radioprotection de l'AP-HM. Elles ont porté sur divers services représentatifs des différents modes d'exposition aux RI en milieu hospitalier, à savoir : cardiologie 
(angio-hémodynamique et électrophysiologie), radiothérapie, médecine nucléaire, radiologie pédiatrique, bloc opératoire pédiatrique, neuro-radiologie et gastroentéro-hépatologie.

Au sein de ces services, nous avons constitué des groupes homogènes d'exposition et étudié les postes de travail représentatifs de ces groupes. Trois types de dosimètres ont été utilisés : des dosimètres opérationnels, des dosimètres photographiques et des pastilles thermoluminescentes (FLi) portées sur le dosimètre opérationnel et le dosifilm dans un objectif comparatif et en différents sites suivant le type d'exposition (poignets, front, thyroïde). Tous ces dosimètres ont été portés sur cinq jours consécutifs de travail et associés à des relevés quotidiens des actes exposant aux RI.

Le bruit de fond a été évalué par des dosimètres opérationnels et thermoluminescents placés dans les lieux de stockage des dosimètres; il a été secondairement soustrait des résultats obtenus sur la semaine d'étude. Enfin, ces résultats ont été extrapolés à 47 semaines de travail annuelles et comparés ainsi aux valeurs limites réglementaires et aux données de dosimétrie passive enregistrées sur les dix dernières années.

\subsubsection{Analyse des données dosimétriques sur dix ans}

À partir des dossiers médicaux, nous avons recueilli et saisi sur informatique les résultats des dosifilms poitrine, et le cas échéant poignet, du personnel inclus dans l'étude, en remontant sur les dix dernières années précédant celle-ci, soit la période [1990-1999].

\subsection{3. Évaluation biogénotoxicologique par le test des micronoyaux}

Cette évaluation, menée en collaboration avec les médecins du travail du personnel hospitalier, a porté sur 132 personnels médicaux et para-médicaux (tous classés en catégorie A) travaillant dans des services de radiothérapie, médecine nucléaire, cardiologie interventionnelle, radiologie et bloc opératoire. Le résultat du test des MN sur lymphocytes a été interprété comparativement à celui obtenu auprès de 69 témoins (personnel administratif hospitalier) appariés avec les exposés sur les critères d'âge, de sexe et de tabagisme. Un complément d'analyse par FISH avec sondes pancentromériques a été réalisé chez deux sous-groupes de 32 exposés et 30 témoins, tirés au sort au sein des populations initiales. Un questionnaire destiné à recueillir des éléments sur d'éventuels facteurs de confusion et sur les caractéristiques de l'exposition professionnelle aux RI ou à d'autres agents physiques ou chimiques a été documenté pour l'ensemble des sujets. 


\subsection{Principaux résultats et discussion}

\subsection{1. Étude des postes de travail avec dosimétrie physique}

Les résultats de ces études de poste, extrapolés à l'année, montrent des doses efficaces corps entier très faibles ; des valeurs de l'ordre de $1 \mathrm{mSv} / \mathrm{an}$ sont fréquemment retrouvées à la console ou à distance du faisceau en salle.

Les doses efficaces les plus élevées sont retrouvées par ordre décroissant :

- en médecine nucléaire : infirmier dans le secteur hospitalisation (13,9 mSv/an), préparation de doses dans le secteur cardiologie du laboratoire (7,6 mSv/an), technicien du laboratoire chaud (6,2 mSv/an);

- en explorations angio-hémodynamiques : cardiologue (4,4 mSv/an).

Les doses efficaces les plus faibles sont relevées au bloc opératoire pédiatrique (mais il est à signaler que l'activité sur la semaine d'étude était plus faible et donc non représentative de l'activité habituelle) et dans le service de radiologie conventionnelle pédiatrique.

Les doses équivalentes les plus élevées sont retrouvées :

- en médecine nucléaire : infirmier dans le secteur hospitalisation (54,7 mSv/an au poignet ; 31,4 mSv/an au front ; 13,4 mSv/an à la thyroïde), préparation de doses dans le secteur cardiologie du laboratoire (39,1 mSv/an au poignet), technicien du laboratoire chaud (23,9 $\mathrm{mSv} / \mathrm{an}$ au poignet) ;

- en explorations angio-hémodynamiques : cardiologue $(83,2 \mathrm{mSv} / \mathrm{an}$ au poignet ; $17,3 \mathrm{mSv} / \mathrm{an}$ au front);

- en gastro-entéro-hépatologie interventionnelle : médecin (67,2 mSv/an au front ; $52,2 \mathrm{mSv} / \mathrm{an}$ au poignet ; 47,7 mSv/an à la thyroïde), infirmier (26,6 mSv/an à la thyroïde) ;

- en neuro-radiologie interventionnelle : médecin (23,6 $\mathrm{mSv} / \mathrm{an}$ au poignet).

Ces résultats sont bien sûr à considérer avec les incertitudes liées à l'extrapolation à l'année (possible défaut de représentativité de la semaine étudiée) et celles liées à la mesure elle-même, estimées à $\pm 20 \%$ pour le dosimètre opérationnel et $\pm 10 \%$ pour les thermoluminescents. Les doses mesurées par les dosimètres thermoluminescents ont été généralement supérieures à celles mesurées par les dosimètres opérationnels ; cela est peut être en rapport avec une meilleure sensibilité du FLi au rayonnement diffusé et une saturation des diodes du dosimètre opérationnel aux forts débits de doses rencontrés en radiologie interventionnelle.

En conclusion, ces études de poste ont permis de proposer de conserver en catégorie A les personnels exposés dans les services suivants : médecine nucléaire 
(hospitalisation, laboratoire, imagerie), cardiologie, gastro-entéro-hépatologie et neuro-radiologie interventionnelles (Bourdieu et al., 2002 ; Bourdieu, 2002).

\subsubsection{Analyse des données dosimétriques sur dix ans}

Sont présentés ici les résultats dosimétriques recueillis pour les 132 personnels de catégorie A ayant participé à l'évaluation biogénotoxicologique par le test des MN. Au total, 1026 dosimétries passives individuelles annuelles ont été analysées sur une période de dix ans. La dosimétrie moyenne corps entier est de $0,17 \mathrm{mSv} \pm 0,47$ $(\min 0-\max 2,60 \mathrm{mSv})$ sur la dernière année $(\mathrm{n}=128)$, de $0,73 \mathrm{mSv} \pm 2,04(\mathrm{~min}$ 0 - $\max 11,4 \mathrm{mSv})$ en dose cumulée sur les cinq dernières années $(\mathrm{n}=106)$ et de $1,66 \mathrm{mSv} \pm 10,18(\min 0-\max 86,15 \mathrm{mSv})$ sur les cinq années précédentes $(n=73)$.

La très grande majorité $(88,9 \%)$ des dosimétries annuelles est inférieure au seuil de détection des dosimètres photographiques (soit 0,2 $\mathrm{mSv}$ à l'époque). Les doses supérieures à ce seuil de détection proviennent des services d'explorations angiohémodynamiques, de médecine nucléaire et de radiothérapie. Seulement 4,2\% (n= 43) des dosimétries enregistrées sont supérieures à $1 \mathrm{mSv} / \mathrm{an}$ et seulement 0,6\% $(\mathrm{n}=6)$ supérieures à $6 \mathrm{mSv} / \mathrm{an}$. Les doses supérieures à $6 \mathrm{mSv}$ (mais inférieures à $20 \mathrm{mSv}$ ) concernent trois personnes : une infirmière du service d'explorations angiohémodynamiques, une infirmière du service de médecine nucléaire et une technicienne du laboratoire de médecine nucléaire. Deux valeurs sont supérieures à $20 \mathrm{mSv}$ (dose limite réglementaire, de $50 \mathrm{mSv} / \mathrm{an}$ alors, dépassée une fois) et concernent un même sujet, cardiologue dans le service d'explorations angiohémodynamiques (Sari-Minodier et al., 2007 ; Sari-Minodier, 2002).

Il est intéressant de moduler ces résultats avec les données concernant les modalités du port des dosimètres relevées dans les questionnaires individuels posés par les médecins du travail aux personnes participant à la biosurveillance. Ainsi $18 \%$ des sujets ont répondu ne jamais porter, ou seulement occasionnellement, leur dosifilm poitrine. De plus, nous avons noté qu'une proportion non négligeable des dosifilms (soit en moyenne $6 \%$ sur la dernière année) n'étaient pas lus (résultats notés « $\mathrm{B} »$ ) car envoyés hors délai à l'organisme de dosimétrie passive. Ces éléments sont bien sûr de nature à entraîner une sous-estimation des dosimétries enregistrées.

\subsection{3. Évaluation biogénotoxicologique par le test des micronoyaux}

Les taux de LBMN dans la population exposée professionnellement aux RI apparaissent statistiquement plus élevés que dans la population témoin (14,9\%० $8,1$ versus $11,8 \%$ o $\pm 6,5 ; p=0,01)$. 
Ces deux populations sont appariées sur les principaux facteurs de confusion connus pour le test des $\mathrm{MN}$, à savoir, le sexe, l'âge et le tabagisme. D'autres facteurs de confusion sont mis en évidence dans cette étude, tels que les antécédents familiaux de cancers et l'irradiation d'origine médicale. Cette dernière, évaluée à partir du nombre et du type d'examens radiologiques médicaux passés sur les trois dernières années et de l'irradiation moyenne produite par chacun de ces examens (données UNSCEAR), parait en effet corrélée aux taux de LBMN alors qu'aucune corrélation n'est mise en évidence entre les taux de LBMN et les dosimétries professionnelles (quasiment toutes inférieures au seuil de détection). Une analyse statistique multivariée, intégrant tous les facteurs de confusion, permet de conclure que le taux de LBMN reste significativement plus élevé chez les exposés comparativement aux témoins, plus élevé également chez les femmes comparativement aux hommes et qu'il augmente avec l'âge ; l'irradiation d'origine médicale et les antécédents médicaux de cancers perdent alors toute significativité statistique (Sari-Minodier et al., 2007 ; Sari-Minodier, 2002).

L'analyse complémentaire en FISH réalisée auprès de sous-groupes de respectivement 32 exposés et 30 témoins, ne permet pas de mettre en évidence de différences qualitatives dans le contenu des MN. En effet, chez les exposés comme chez les témoins, les MN observés, pour 2/3 d'entre eux, sont centromère-positifs. Ils contiennent donc majoritairement un ou plusieurs chromosomes entiers et sont consécutifs à des évènements aneugènes.

En conclusion, les situations d'exposition professionnelle aux faibles doses de RI en milieu hospitalier, telles qu'elles ont été documentées dans ces travaux, conduisent à une augmentation des dommages chromosomiques dans les lymphocytes des sujets concernés. Il faut souligner que le test des MN ne constitue pas un biomarqueur spécifique de tel ou tel génotoxique de l'environnement. Il intègre l'action des différents agents clastogènes et/ou aneugènes présents dans l'environnement professionnel mais également extra-professionnel. Néanmoins, le résultat du biomarqueur peut être raisonnablement attribuée à l'environnement professionnel dans la mesure où la population témoin est choisie pour être similaire à la population exposée, en ce qui concerne les expositions extraprofessionnelles et les facteurs individuels intrinsèques. Quant à l'environnement professionnel, il est susceptible de comporter, outre les RI, d'autres agents potentiellement génotoxiques. Au vu des très faibles doses de RI enregistrées chez le personnel soignant, les dommages chromosomiques observés pourraient d'ailleurs être en rapport avec des multi-expositions qui restent à documenter avec plus de précisions. Il n'est pas possible non plus d'exclure, dans cette population de personnel hospitalier, le rôle éventuel de l'irradiation d'origine médicale, malgré l'absence de significativité statistique en analyse multivariée. 
Les taux de MN semblent bien être le résultat combiné de l'association de l'environnement et du statut génétique de l'individu. Toutefois, les résultats de ce biomarqueur ne peuvent être interprétés qu'au niveau collectif et aucune extrapolation ne peut être faite à l'échelle individuelle, compte tenu notamment des très nombreuses incertitudes concernant les facteurs de susceptibilité. La signification, en terme de risque pour la santé, d'un taux élevé de MN au niveau d'un groupe n'est pas clairement établie à ce jour. L'intérêt de ce biomarqueur se situe bien dans le domaine de la prévention. Il ne peut se concevoir qu'intégré au sein d'une démarche globale de prévention du risque cancérogène professionnel et apparaît complémentaire des autres moyens d'identification des dangers et d'évaluation des risques. Il permet en effet de nous informer de la présence de modifications biologiques contemporaines de l'exposition, ce qui doit aboutir à la mise en œuvre précoce de mesures de prévention adéquates, d'autant plus efficaces qu'elles s'adressent à une pathologie différée. Outre les mesures techniques, organisationnelles et médicales, il convient de souligner l'importance de l'information et de la sensibilisation des personnels exposés, en tant que principaux acteurs de la réduction du risque radiologique et de la fidélité de son évaluation dosimétrique.

\section{Conclusions et perspectives}

Les études de caractérisation des expositions et de détection précoce des effets génotoxiques des faibles expositions aux RI présentées ci-dessus sont bien en cohérence avec les objectifs spécifiés dans l'introduction générale. Il apparaît notamment l'apport déterminant de la dosimétrie opérationnelle en temps réel, qui a pour objectif l'approche individuelle et en situation de travail du niveau d'exposition. En outre, le caractère non spécifique de la plupart des tests, tels que la numération des $\mathrm{MN}$ utilisés dans la biosurveillance, ajoute de l'intérêt à l'approche dosimétrique qui, par son caractère spécifique et au plus près du salarié, permet d'apprécier la réalité de l'exposition professionnelle.

Les perspectives en terme d'amélioration de la caractérisation des expositions doivent intégrer deux aspects, d'une part l'aspect dosimétrie physique avec l'amélioration des modèles prévisionnels d'exposition et des performances des dosimètres individuels, et d'autre part l'aspect biosurveillance avec l'évolution de biomarqueurs plus spécifiques de l'impact des RI. Ce dernier aspect fait d'ailleurs aujourd'hui l'objet de recherches cytogénétiques et toxicogénomiques prometteuses. Enfin, la prévention des risques, pour être véritablement efficace doit passer par une amélioration de l'information des salariés et du grand public pour éviter les dérives aussi bien vers un excès d'alarmisme que vers le désintérêt. 
Remerciements : au personnel technique et aux stagiaires du laboratoire BME ; aux médecins du travail impliqués dans la biosurveillance, aux membres des groupes de travail « radiographie industrielle » et à la cellule de radioprotection de l'AP-HM ; à la DRTE-FP PACA, au Ministère de l'emploi et de la solidarité, à l'Association pour la recherche sur le cancer et la Fondation Philippe Daher (Fondation de France) pour leur soutien financier.

\section{RÉFÉRENCES}

Azzopardi G. (2005) Évaluer et prévenir le risque radiologique professionnel dans les opérations de radiographie industrielle, Rapport de stage BTS Radioprotection, INSTN Cadarache.

Azzopardi G., Klemenic O., Coletti F., Paul D. (2005) Analyse de l'exposition, optimisation de la dosimétrie en radiographie industrielle, XXII ${ }^{\mathrm{es}}$ Journées des LARD, Montbéliard.

Botta C., Iarmarcovai G., Chaspoul F., Sari-Minodier I., Pompili J., Orsière T., Bergé-Lefranc J.L., Botta A., Gallice P, De Méo M. (2006) Assessment of occupational exposure to welding fumes by inductively coupled plasma-mass spectroscopy and by the alkaline Comet assay, Environ. Mol. Mutagen. 47, 284-295.

Bourdieu A.L. (2002) Étude préliminaire à la mise en place de la dosimétrie opérationnelle à l'hôpital, Thèse de doctorat de Médecine, Faculté de Médecine de Marseille, université de la Méditerranée.

Bourdieu A.L., Sari-Minodier I., Botta A. (2002) Évaluation de l'exposition du personnel soignant aux rayonnements ionisants par dosimètres opérationnels et pastilles thermoluminescentes. Dans : $27^{e}$ Congrès National de Médecine du Travail, 4-7 juin 2002, Grenoble, numéro spécial des Arch. Mal. Prof. 63, 200.

Coletti F., Paul D., Sari-Minodier I., Botta A. (2006) Risk assessment at worplaces in industrial radiography: from research to action, Second European IRPA Congress on Radiation Protection, Paris.

Decome L., De Méo M., Geffard A., Doucet O., Duménil G., Botta A. (2005) Evaluation of photolyase (Photosome) repair activity in human keratinocytes after a single dose of ultraviolet B irradiation using the comet assay, J. Photochem. Photobiol. 79, 101-108.

Iarmarcovai G., Sari-Minodier I., Chaspoul F., Botta C., De Méo M., Orsière T., Bergé-Lefranc J.L., Gallice P., Botta A. (2005) Risk assessment of welders using analysis of eight metals by ICPMS in blood and urine and DNA damage evaluation by the comet and micronucleus assays; influence of XRCC1 and XRCC3 polymorphisms, Mutagenesis 20, 425-32.

Klemenic O. (2005) Le risque professionnel en radiographie industrielle : éléments nécessaires pour la nouvelle charte de bonnes pratiques, Rapport de stage d'élève ingénieur, ESAIP.

Orsière T., De Méo M., Sari-Minodier I., Botta A. (2000) Contribution des tests à court terme dans l'évaluation du potentiel cancérogène d'une substance ou d'un mélange de substances. Dans : Les cancers professionnels Tome I (J.C. Pairon, P. Brochard, J.P. Le Bourgeois, P. Ruffié, Eds) pp. 141-157. Éditions Margaux Orange, Paris.

Orsière T., De Méo M. (2005) Génotoxicité et Mutagenèse. Dans : Toxicologie (A. Viala, A. Botta, Eds) pp. 25-46. Éditions Lavoisier, Paris.

Paul D., Pizzorno M., Abela G., Gschwind R., Provens H., Plé J., Sari-Minodier I., Coletti F. (2006) Analyse de l'exposition et optimisation de la dosimétrie en radiographie industrielle. Dans : $4^{e s}$ journées SFRP sur l'optimisation de la radioprotection dans les domaines électronucléaire, industriel et médical, 26\&27 septembre 2006, La Rochelle. 
Pizzorno M., Coletti F., Paul D., Sari-Minodier I., Azzopardi G., Klemenic O., Botta A. (2006) Évaluation et prévention des risques professionnels dans les opérations de radiographie industrielle, $\lambda \mu 15,15^{e}$ congrès de Maîtrise des Risques et de Sûreté de Fonctionnement, Lille.

Sari-Minodier I., Orsière T., Charrier D., Botta A. (2000) Génotoxicité des rayonnements ionisants chez les radiologues industriels. Dans : $21^{e}$ congrès de l'ATSR (Association pour les Techniques et Sciences de Radioprotection), 25-27 octobre 2000, Aix-en-Provence.

Sari-Minodier I. (2002) Évaluation biogénotoxicologique des expositions professionnelles aux rayonnements ionisants dans les secteurs de la santé et de la radiographie industrielle, Thèse de l'université de la Méditerranée.

Sari-Minodier I., Orsière T., Bellon L., Pompili J., Sapin C., Botta A. (2002) Cytogenetic monitoring of industrial radiologists using the micronucleus assay, Mutat. Res. 521, 37-46.

Sari-Minodier I., Orsière T., Botta A. (2004) Quantification des effets biologiques des faibles doses de rayonnements ionisants dans une population de radiologues industriels. Dans : $28^{e}$ Congrès National de Médecine du Travail, 8-11 juin 2004, Bordeaux, numéro spécial des Arch. Mal. Prof. 65, 267.

Sari-Minodier I., Orsière T., Auquier P., Martin F., Botta A. (2007) Cytogenetic monitoring by the use of the micronucleus assay among hospital workers exposed to low doses of ionizing radiation, Mutat. Res. 629, 111-121. 